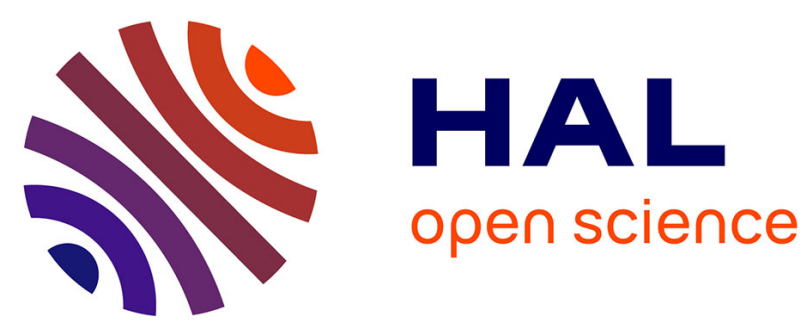

\title{
Dynamics of the stress-mediated magnetoelectric memory cell $\mathrm{Nx}$ (TbCo2/FeCo)/PMN-PT
}

Vladimir Preobrazhensky, Alexey Klimov, Nicolas Tiercelin, Yannick Dusch, Stefano Giordano, Anton Churbanov, Theo Mathurin, Philippe Pernod, Alexander Sigov

\section{To cite this version:}

Vladimir Preobrazhensky, Alexey Klimov, Nicolas Tiercelin, Yannick Dusch, Stefano Giordano, et al.. Dynamics of the stress-mediated magnetoelectric memory cell $\mathrm{Nx}(\mathrm{TbCo} / \mathrm{FeCo}) / \mathrm{PMN}-\mathrm{PT}$. Journal of Magnetism and Magnetic Materials, 2018, 459, pp.66-70. 10.1016/j.jmmm.2017.12.028 . hal02318299

\section{HAL Id: hal-02318299 \\ https://hal.science/hal-02318299}

Submitted on 24 Sep 2020

HAL is a multi-disciplinary open access archive for the deposit and dissemination of scientific research documents, whether they are published or not. The documents may come from teaching and research institutions in France or abroad, or from public or private research centers.
L'archive ouverte pluridisciplinaire HAL, est destinée au dépôt et à la diffusion de documents scientifiques de niveau recherche, publiés ou non, émanant des établissements d'enseignement et de recherche français ou étrangers, des laboratoires publics ou privés. 


\title{
Dynamics of the stress-mediated magnetoelectric memory cell $\mathrm{N} \times\left(\mathrm{TbCo}_{2} / \mathrm{FeCo}\right) / \mathrm{PMN}-\mathrm{PT}$
}

\author{
Vladimir Preobrazhensky ${ }^{\mathrm{a}, \mathrm{b}}$, Alexey Klimov ${ }^{\mathrm{c}, \mathrm{d}}$, Nicolas Tiercelin ${ }^{\mathrm{b}}$, Yannick Dusch $^{\mathrm{b}}$, Stefano Giordano $^{\mathrm{b}}$, Anton $_{\text {Churbanov }}^{\mathrm{d}, \mathrm{e}}$, \\ Theo Mathurin ${ }^{\mathrm{b}}$, Philippe Pernod ${ }^{\mathrm{b}}$, Alexander Sigov ${ }^{\mathrm{c}}$

\footnotetext{
${ }^{a}$ Joint International Laboratory LIA LICS: Wave Research Center, A.M.Prokhorov GPI, RAS, ul. Vavilova 38, Moscow, 119991 Russia

${ }^{b}$ Joint International Laboratory LIA LICS: Univ. Lille, CNRS, Centrale Lille, ISEN, Univ. Valenciennes, UMR 8520 - IEMN, F-59651 Lille, France

${ }^{c}$ Joint International Laboratory LIA LICS: Moscow Technological University (MIREA) Vernadsky Avenue 78, Moscow, 119454 Russia
} \\ ${ }^{d}$ Joint International Laboratory LIA LICS: V. A. Kotel'nikov Inst. of Radioeng. and Electronics (IRE RAS), ul. Mokhovaya 11/7, Moscow, 125009 Russia \\ ${ }^{e}$ Moscow Institute of Physics and Technology, Dolgoprudny, Institutskiy lane, 9, 141700, Russia
}

\begin{abstract}
Stress-mediated magnetoelectric heterostructures represent a very promising approach for the realization of ultra-low energy Random Access Memories. The magnetoelectric writing of information has been extensively studied in the past, but it was demontrated only recently that the magnetoelectric effect can also provide means for reading the stored information. We hereby theoretically study the dynamic behaviour of a magnetoelectric random access memory cell (MELRAM) typically composed of a magnetostrictive multilayer $\mathrm{N} \times\left(\mathrm{TbCo}_{2} / \mathrm{FeCo}\right)$ that is elastically coupled with a $<011>\mathrm{PMN}-\mathrm{PT}$ ferroelectric crystal and placed in a Wheatstone bridge-like configuration. The numerical resolution of the LLG and electrodynamics equation system demonstrates high speed write and read operations with an associated extra-low energy consumption. In this model, the reading energy for a 50nm cell size is estimated to be less than $5 \mathrm{aJ} / \mathrm{bit}$.
\end{abstract}

Keywords: Magnetoelectric Memory, MELRAM, Extra-low energy, Magnetoelectric readout, Straintronics

\section{Introduction}

The new generation of data storage technology requires high speed, high density, low power and nonvolatile random access memory (RAM) [1, 2]. A very promising approach for energy efficient RAMs is based on the magneto-electric (ME) interaction in nano-composite multiferroic structures [3-9]. The ME interaction in such structures lies in the elastic coupling of magnetostrictive and piezoelectric layers [10] that can be chosen to operate efficiently at room temperature. In several works, the high efficiency of this concept was demonstrated [11,12] and, in particular, the energy consumption for sub-nanosecond writing of one bit in a nanoscale strain-mediated magneto-electric memory (MELRAM) cell based on $\mathrm{N} \times\left(\mathrm{TbCo}_{2} / \mathrm{FeCo}\right) / \mathrm{PZT}$ structure was estimated to be of a few tenths of femtoJoules [13-15]. However, the problem of reading the information from the MELRAM is a challenging problem, still under investigation. One class of solutions assume that the readout will be operated through spintronic devices, either using magnetic tunnel junctions (MTJ) [16-19] or giant magnetoresistance (GMR) stacks [20]. However, such approaches not only require straintronic-spintronic technology compatibility, but they also need the development of quite complicated heterostructures. In Ref.[21], we recently proposed and experimentally demonstrated the use of the ME effect for writing as well as reading information in a MELRAM macroscopic prototype based on a $\mathrm{N} \times\left(\mathrm{TbCo}_{2} / \mathrm{FeCo}\right) / \mathrm{PMN}-\mathrm{PT}$ heterostructure. In the present paper, we hereby study the complete writing/reading dynamics of such a memory cell at submicron and nanometric scales. This study is carried out by numerical simulations using the cou-

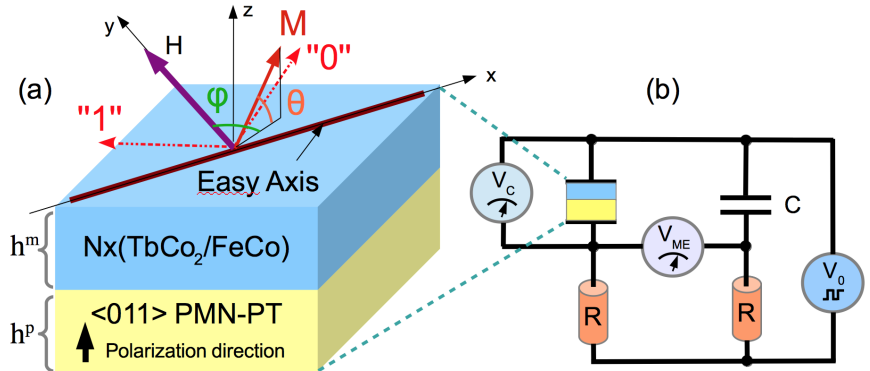

Figure 1: a) Schematic representation of the stress-mediated MELRAM cell. Two stable orientations of magnetization M are: $\varphi_{0}= \pm \pi / 4, \theta=0$; b) Electric circuit for the magnetoelectric readout of information stored in the magnetic subsystem of MELRAM.

pled system of Landau-Lifshitz-Gilbert (LLG) equation for the magnetic subsystem and electrodynamic equations for describing the electric circuit containing the MELRAM cell.

\section{Constitution and mathematical model of the MELRAM cell}

The memory cell under consideration is presented in Fig.1. The typical involved materials are a rare-earth multilayer with giant magnetostriction and uniaxial in-plane magnetic anisotropy that consists of $\mathrm{N}$ nano-bilayers $\mathrm{TbCo}_{2} / \mathrm{FeCo}$, deposited on top of a $<011>$ cut PMN-PT ferroelectric crystal having a polarization axis normal to the structure plane $[3,4,22,23]$. The details of the $\mathrm{N} \times\left(\mathrm{TbCo}_{2} / \mathrm{FeCo}\right)$ multilayer technology are described in Refs. [24, 25]. The structure is 
placed in a DC magnetic field $H$ in the plane of the film, normal to the easy anisotropy axis (EA), i.e. along the y-axis. Assuming an homogeneous magnetization, the energy density $F_{m}$ of the magnetic sub-system contains Zeeman, anisotropy and demagnetizing components and can be expressed as follows in the chosen cartesian coordinates system:

$$
F_{m}=-M_{y} H-\frac{H_{A}}{2 M} M_{x}^{2}+2 \pi M_{z}^{2}
$$

where $\vec{M}=\left(M_{x}, M_{y}, M_{z}\right)$ is the magnetization vector and $H_{A}$ is the uniaxial anisotropy field. When the magnetic field $H$ is smaller than $H_{A}$ the magnetic system is bistable. Two energetically equivalent in-plane orientations with angles $\pm \varphi_{0}$ relatively to $H$ are indeed available for the magnetization. These two equilibrium orientations can be associated with bit values " 1 " and " 0 ", respectively. When $H=\frac{H_{A}}{\sqrt{2}}\left(\varphi_{0}=\pi / 4\right)$ the angle between the equilibrium orientations is equal to $\pi / 2$ (perpendicular states). The application of a voltage $V_{C}(t)$ to the PMN-PT layer induces an in-plane anisotropic mechanical deformation of the structure and can result in the switch of magnetization from one equilibrium state to another. This switch corresponds to a voltage-induced writing of information in the magnetic subsystem. The energy of the stress-mediated magnetoelectric interaction in the model of an effective medium was derived in Ref. [21] as:

$$
F_{M E}=\sigma V_{c}(t) M_{x} M_{y}
$$

where $\sigma$ is the magnetoelectric parameter:

$$
\sigma=\frac{B\left(d_{31}-d_{32}\right)}{M^{2}\left(h^{p}+h^{m}\right)}
$$

Here, $B$ is the magnetostriction constant of the magnetic layer, $d_{i j}$ are the piezoelectric parameters of the ferroelectric crystal, $h^{m}$ and $h^{p}$ are the thicknesses of magnetic and ferroelectric layers respectively and $M$ is the saturation magnetization. The dynamics of the magnetization switch under an applied voltage is described by the Landau-Lifshitz-Gilbert (LLG) equation:

$$
\frac{d \vec{M}}{d t}=-\gamma \vec{T}+\frac{\alpha}{M}\left(\vec{M} \wedge \frac{d \vec{M}}{d t}\right)
$$

where $\gamma$ is the magneto-mechanical ratio and $\alpha$ is the relaxation parameter. The torque vector $\vec{T}$ is equal to:

$$
\vec{T}=-\vec{M} \wedge \frac{\partial\left(F_{m}+F_{M E}\right)}{\partial \vec{M}}
$$

For a convenient description of the nonlinear dynamics, the angular representation of the LLG equation is introduced by means of the following relations:

$$
M_{x}=M \cos \theta \sin \varphi, M_{y}=M \cos \theta \cos \varphi, M_{z}=M \sin \theta, \text { (6) }
$$

where $\varphi$ and $\theta$ are the in-plane and out-of-plane magnetization angles, respectively, as shown on Fig.1a. The angular form of the LLG equation corresponds to the system of equations:

$$
\begin{aligned}
& \frac{d \varphi}{d t}=-\frac{\gamma}{\Delta}\left[T_{x} \cos \theta+T_{z}(\sin \theta \sin \varphi-\alpha \cos \varphi)\right] \\
& \frac{d \theta}{d t}=-\frac{\gamma}{\Delta}\left[T_{x} \alpha \cos ^{2} \theta+T_{z}(\cos \theta \cos \varphi+\alpha \sin \theta \cos \theta \sin \varphi)\right],
\end{aligned}
$$

where

$$
\begin{aligned}
\Delta=(1+ & \left.\alpha^{2}\right) \cos ^{2} \theta \cos \varphi \\
T_{x}= & -2 \pi M \sin (2 \theta) \cos \varphi-H \sin \theta \\
& +\frac{1}{2} \sigma V_{c}(t) M \sin (2 \theta) \sin \varphi \\
T_{z}= & H \cos \theta \sin \varphi-\frac{1}{2} H_{A} \cos ^{2} \theta \sin (2 \varphi) \\
& +\sigma V_{c}(t) M \cos (2 \varphi) \cos ^{2} \theta .
\end{aligned}
$$

Since the magnetoelastic layer is mechanically coupled to the ferroelectric crystal, the dynamic reorientation of the magnetization induces variations of the electric polarization in the ferroelectric subsystem. This is the crucial point of the magnetoelectric readout principle. According to Ref. [21], the magnetoelectric component of the polarization is equal to:

$$
P_{M E}=\frac{h^{m}}{2\left(h^{p}+h^{m}\right)} B\left(d_{31}-d_{32}\right) \sin (2 \varphi)
$$

$P_{M E}$ being a function of $\varphi$, it follows that the measure of this 'magnetoelectric' polarization can be used for the determination of the magnetic state of the structure and thus provides a readout method of the information stored in the magnetic subsystem. In order to accurately describe both writing and readout (W/R) operations, the LLG system of equations (7) has to be completed by the electrodynamic equations for the electrical circuit in which the magnetoelectric cell is placed. As a matter of fact, the simplest circuit to consider is a Wheatstone bridge, as shown schematically in Fig.1b. Of course, from the technological point of view, the consideration of the Wheatstone bridge leads to the occupation of a given area on the memory chip. This point may generate a limitation concerning the integration density. In the proposed scheme, the voltage $V_{C}$ applied to the structure and the magnetoelectric signal $V_{M E}$ across the diagonal of the bridge are described by the equations:

$$
R C \frac{d V_{c}}{d t}+V_{c}+R S \frac{d P_{M E}}{d t}=V_{0}(t)
$$

and

$$
R C \frac{d V_{M E}}{d t}+V_{M E}+R S \frac{d P_{M E}}{d t}=0
$$

where $R$ is the resistance of the bridge, $C$ and $S$ are respectively the structure capacitance and cross-section, and $V_{0}(t)$ is the applied $\mathrm{W} / \mathrm{R}$ control voltage. The complete set of equations (7),(11) and (12) was solved using a commercial mathematical simulation software for typical W/R modes. The results are presented and discussed in the following section.

\section{Results and discussion}

A first set of simulations was carried out on a 500nm wide cell which, from a technological point of view, is a realistic scale for our future sub-micron prototypes and is small enough for the demonstration of their typical dynamic behaviour and 
energy efficiency. The results of further downscaling are discussed at the end of this section. The following geometry of the cell is assumed: the cross-section area is $500 \mathrm{~nm} \times 500$ $\mathrm{nm}$, the PMN-PT layer is $h_{p}=500 \mathrm{~nm}$ thick and the magnetostrictive material is a $25 \times\left(\mathrm{TbCo}_{2(4 \mathrm{~nm})} / \mathrm{FeCo}_{(4 \mathrm{~nm})}\right)$ multilayer with a total thickness of $h_{m}=200 \mathrm{~nm}$. The typical magnetic parameters for this multilayer are: $M=1000 \mathrm{emu} / \mathrm{cm}^{3}, B=10$ $\mathrm{MPa}, H_{A}=1000 \mathrm{Oe}$, relaxation parameter $\alpha=0.1$ and applied static field $H=780$ Oe. The parameters of the $<011>$ PMN-PT crystal are : $\varepsilon_{33}=4000, d_{31}-d_{32}=2.5 \times 10^{-9} \mathrm{C} / \mathrm{N}$ [26]. Given the value of $\varepsilon_{33}$, the capacitance $C$ of the cell due to the PMNPT dielectric permittivity can be estimated and is about $18 \mathrm{fF}$. This value is chosen for the balance capacitance on the right hand of Fig.1b. The balance resistance is chosen equal to $R=30$ $k \Omega$. The sequence of pulses for the control voltage $V_{0}(t)$ is shown in Fig.2a using the dimensionless time scale $\gamma M t$, where $\gamma M=17.6 \times 10^{9} s^{-1}$.

The results of the simulations are presented on Fig.2b-d and Fig.3. Fig.2b illustrates the magnetization switch between the equilibrium positions " 1 " and " 0 ". The phase trajectories of the magnetic moment during the switch processes (i.e. " $1 " \rightarrow " 0$ " and $" 0 " \rightarrow " 1 ")$ are shown on Fig.3. During the reorientation, the magnetization slightly leaves the plane of the structure as it was mentioned in Ref. [13].

In both cases, the magnetization switch is accompanied by the appearance of an electric pulse $V_{M E}$ of about $80 \mathrm{mV}$ across the bridge diagonal (see Fig.2c) with a sign depending on the direction of the switch. On Fig.2c, the appearance of the $V_{M E}$ pulse during the first positive $V_{0}$ driving pulse indicates an initial state as "1". Upon the application of a second positive driving pulse, no $V_{M E}$ signal is measured, indicating the " 0 " state. On the other hand, the application of a negative driving pulses sequence induces an inversed process. The detection of the $V_{M E}$ signal upon application of a unipolar control pulse $V_{0}$ is thus a new readout principle for the information stored in magnetoelectric memories. Besides the fact that it removes the need for complex magnetoresistive structures, it also exhibits a good energy efficiency, which is comparable to that of magnetoelectric writing using electric field pulses [15]. A drawback of this approach is that the readout is destructive, since the applied control pulse may induce the magnetization switch. But the memory state can automatically be restored using the generated output electric signal $V_{M E}$ to trigger a writing pulse with an opposite polarity and a proper delay. As it follows from the data in Fig.2, the magnetization switch arises in about $1.5 \mathrm{~ns}$ after beginning of the control pulse. This delay is actually due to the necessary time for the electric field to reach the threshold strength in the ferroelectric layer for the magnetization switch. The evolution of the $V_{c}(t)$ voltage across the magnetoelectric memory cell is shown in Fig.2d where magnetoelectric peaks are clearly visible when the magnetization switches occur. The time delay is large enough to ensure the separation of reading and restoration control pulses and thus the automatic restoration of the initial magnetic state.

Some assumptions used in this model must be justified as follows. First of all, we neglected the modeling of the elastodynamics within the system. It means that the mechanical
Table 1: Parameters of the MELRAM cell obtained by numerical simulations for different cell sizes.

\begin{tabular}{|c|c|c|c|}
\hline Parameter & "500" & "100" & "50" \\
\hline $\mathrm{S}\left(\mathrm{nm}^{2}\right)$ & $500 \times 500$ & $100 \times 100$ & $50 \times 50$ \\
\hline $\mathrm{h}^{\mathrm{p}}(\mathrm{nm})$ & 500 & 100 & 50 \\
\hline $\mathrm{h}^{\mathrm{m}}(\mathrm{nm})$ & 200 & 100 & 50 \\
\hline $\mathrm{C}(\mathrm{fF})$ & 18 & 3.6 & 1.8 \\
\hline $\mathrm{R}(\mathrm{k} \Omega)$ & 30 & 50 & 90 \\
\hline $\mathrm{V}_{0}(\mathrm{mV})$ & 200 & 100 & 50 \\
\hline $\mathrm{t}_{s}(\mathrm{~ns})$ & 1.7 & 0.28 & 0.48 \\
\hline $\mathrm{V}_{M E}(\mathrm{mV})$ & 80 & 23 & 13 \\
\hline $\mathrm{E}$ (aJ/bit) & 700 & 36 & 4.5 \\
\hline
\end{tabular}

waves propagation has not been considered during the transitional phases. This point is justified by the fact that the magnetic switching time $(1.7 \mathrm{~ns}$ for the cell $500 \mathrm{~nm} \times 500 \mathrm{~nm})$ is much larger than $t_{0}=1 / f_{0}$, where $f_{0}$ is the elastic eigenfrequency of the particle. Since we can approximate $f_{0}$ with $v / d$, where $v$ is the elastic wave velocity and $d$ is the typical size of the cell, we have that $t_{0} \sim 3 \times 10^{-10} \mathrm{~ns}$, thus confirming our hypothesis.

Moreover, we supposed to deal with a monodomain magnetic system, i.e. with all spins behaving collectively (macrospin formulation). This assumption, which could be not fulfilled for the large cells, can be typically checked by comparing the magnetic energy of a uniformly magnetized particle with the exchange energy of a multidomain particle [27]. In our case this analysis leads to a critical size of a few tens of nanometers. However, the nearly complete absence of domains generation within the two stable states has been proved by different experimental characterizations in previous works [4]. Briefly, this point has been verified by observing that two stable states correspond to the saturation region of the magnetization curve. We may suppose the formation of a multidomain structure during the transitional dynamic phases, but this point do not alter the operation of the memory element.

Also, the thermal effects should be considered in order to prove the stability of the magnetic states. Thermal fluctuations within the magnetoelastic particles were studied in Ref.[14, 28]. It was shown that thermal effects are controlled by the ratio of the thermal energy $\left(k_{B} T\right)$ to the particle volume $(V)$. When this ratio is smaller than $10^{3} \mathrm{~J} / \mathrm{m}^{3}$ the thermal effects are proved to be negligible. For the system under consideration the maximal ratio is $k_{B} T / V=40 \mathrm{~J} / \mathrm{m}^{3}$. Concerning the thermal fluctuations generated by the RC circuit, we can mention that the Nyquist noise mean-square voltage $\left\langle u^{2}\right\rangle=k_{B} T / C$ is much smaller than the square of $V_{0}$, required to switch the magnetic moment. Indeed, in our case we have $V_{0}^{2} /<u^{2}>\sim 80$, which correspond to an error probability smaller than $10^{-17}$.

The simulated characteristics of the $500 \mathrm{~nm}$ cell as well as for two similar cells with further downscaling are summarized in Table 1, where $S$ is the cell area, $t_{S}$ is the W/R time and $E$ is the energy consumption due to the charge-discharge of the cell ca- 


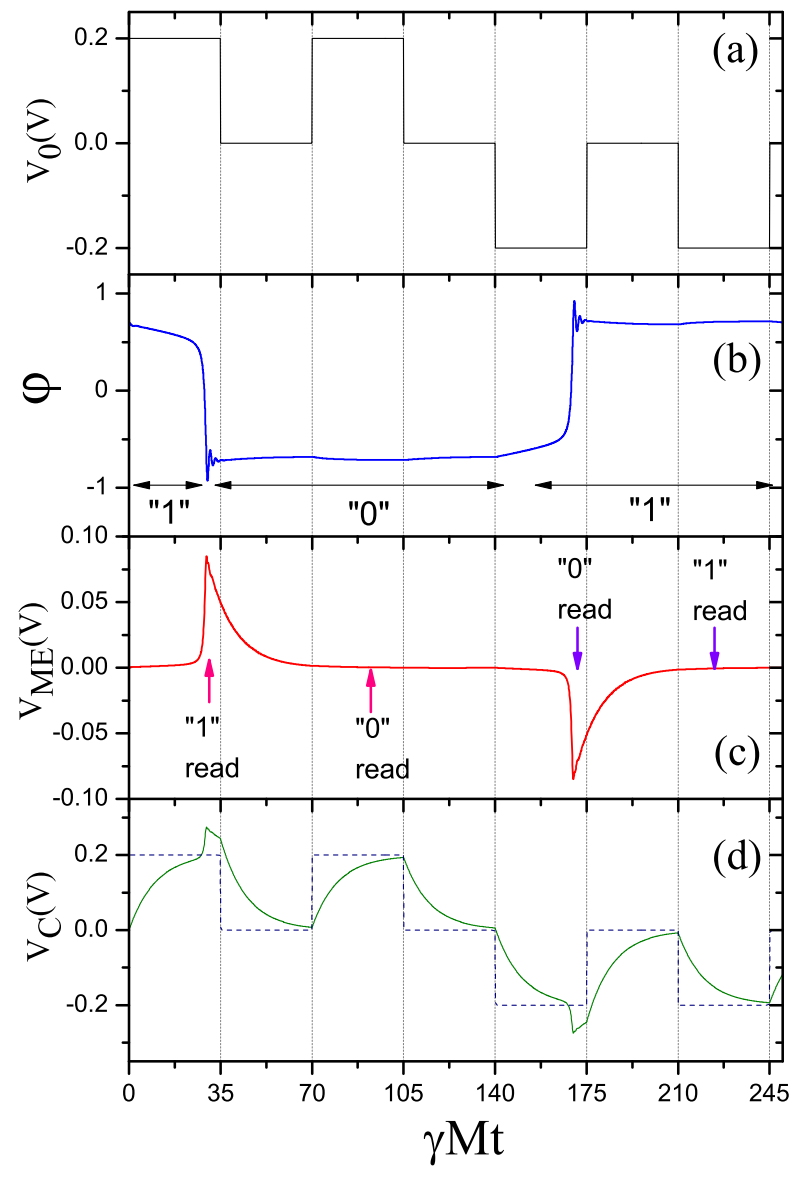

Figure 2: Dynamics of writing and readout in a MELRAM cell: a) Control pulse sequence; b) Voltage induced magnetization switch; c) magnetoelectric readout signal; d) Voltage across the magnetoelectric memory cell.

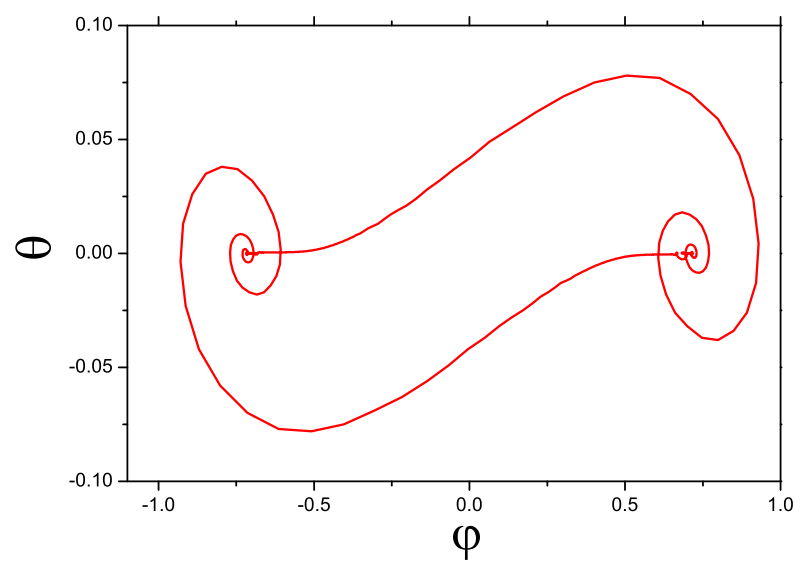

Figure 3: Phase diagram of the magnetization during transitions between the two stable positions. pacity during the $\mathrm{W} / \mathrm{R}$ processes. It shows that the $\mathrm{W} / \mathrm{R}$ voltage $V_{0}$ and magnetoelectric signal $V_{M E}$ decrease with downscaling, but the ratio $V_{M E} / V_{0}$ remains higher than $20 \%$. The choice of the resistance $R$ is guided by a compromise between the $V_{M E}$ value and the $\mathrm{W} / \mathrm{R}$ time $\mathrm{t}_{S}$. Increasing $R$ enhances the $V_{M E}$ signal but also increases $\mathrm{W} / \mathrm{R}$ time. The decrease of the control voltage $V_{0}$ and the cell capacity $\mathrm{C}$ results in a significant gain in the energy consumption $E$, which is due to the dissipated Joule heat during the charge/discharge of the capacitor formed by the ferroelectric material. For the smallest cell, an extra-low energy consumption of a few attoJoule per bit is achieved.

It is advisable to compare the obtained results with the performance of a typical $45 \mathrm{~nm}$ Spin Torque Transfer Magnetic memory (STT-RAM) [29, 30] based on a Magnetic Tunnel Junction (MTJ) with a resistance-area product of $10 \Omega \mu \mathrm{m}^{2}$ that corresponds to a MTJ resistance of $5 \mathrm{k} \Omega$. For sensing currents in the lowest end of the required values, that is to say $10 \mu \mathrm{A}$, and a readout time of $1 \mathrm{~ns}$, the reading energy lost in Joule heating will be of $5 \times 10^{-16} \mathrm{~J} / \mathrm{bit}$ which is comparable with the energy consumption of the 500nm MELRAM and two order of magnitude worse than what could potentially be achieved using a $50 \mathrm{~nm}$ MELRAM. It is worth to mention that a recently introduced technique, based on the separation of read and write units in multiferroic devices, resulted in a theoretical dissipation of $1 \mathrm{aJ}$ [18] or $0.1 \mathrm{aJ}$ [19] for one cycle of the memory operation. This approach is based on the use of MTJ structures and gives ultralow energy consumptions needing, however, a more elaborated nanotechnological design.

\section{Conclusions}

The numerical simulations we described demonstrate the efficiency of a new principle for the magnetoelectric writing and reading in stress-mediated composite multiferroic memory cells. The information stored in the magnetic subsystem of the cell is probed with the application of a low voltage ranging from $200 \mathrm{mV}$ down to $50 \mathrm{mV}$ depending on the cell size. The corresponding output readout signal ranges from $80 \mathrm{mV}$ down to $13 \mathrm{mV}$ and the writing/reading time ranges from $1.7 \mathrm{~ns}$ downto $0.48 \mathrm{~ns}$. The expected energy consumption is extra-low and strongly dependent on the cell size scale. For cells with a decreasing size from 500 down to $50 \mathrm{~nm}$, this amount of energy varies from $700 \mathrm{aJ} / \mathrm{bit}$ down to only $4.5 \mathrm{aJ} / \mathrm{bit}$. These features finally prove that our proposed system is strongly competitive with existing technologies, also from the point of view of the structure simplicity.

\section{Acknowledgements}

The work was supported by the grant RFBR 16-29-14022 and the StartAirr program MELRAM of the région Hauts de France. Technological developments are supported by the french RENATECH network.

[1] ITRS, Emerging research devices, Tech. rep., THE INTERNATIONAL TECHNOLOGY ROADMAP FOR SEMICONDUCTORS (2013).

URL http://www.itrs.net/Links/2013ITRS/2013Chapters/ 2013ERD.pdf 
[2] A. Chen, A review of emerging non-volatile memory (nvm) technologies and applications, Solid-State Electronics 125 (2016) 25-38.

URL http://ww.sciencedirect.com/science/article/pii/ S0038110116300867

[3] N. Tiercelin, Y. Dusch, V. Preobrazhensky, P. Pernod, Magnetoelectric memory using orthogonal magnetization states and magnetoelastic switching, J. Appl. Phys. 109 (7) (2011) 07D726-3.

URL http: //link. aip.org/link/?JAP/109/07D726/1

[4] N. Tiercelin, Y. Dusch, A. Klimov, S. Giordano, V. Preobrazhensky, P. Pernod, Room temperature magnetoelectric memory cell using stressmediated magnetoelastic switching in nanostructured multilayers, Appl. Phys. Lett. 99 (19) (2011) 192507-3.

URL http: //link. aip.org/link/?APL/99/192507/1

[5] J.-M. Hu, Z. Li, L.-Q. Chen, C.-W. Nan, High-density magnetoresistive random access memory operating at ultralow voltage at room temperature, Nature Communications 2 (2011) 553-.

URL http: //dx.doi.org/10.1038/ncomms 1564

[6] T. Wu, A. Bur, K. Wong, P. Zhao, C. S. Lynch, P. K. Amiri, K. L. Wang, G. P. Carman, Electrical control of reversible and permanent magnetization reorientation for magnetoelectric memory devices, Applied Physics Letters 98 (26) (2011) 262504, journal article.

URL http://dx.doi.org/doi/10.1063/1.3605571

[7] M. Ghidini, R. Pellicelli, J. Prieto, X. Moya, J. Soussi, J. Briscoe, S. Dunn, N. Mathur, Non-volatile electrically-driven repeatable magnetization reversal with no applied magnetic field, Nature Communications 4 (2013) 1453-.

URL http: //dx .doi.org/10.1038/ncomms 2398

[8] A. K. Biswas, S. Bandyopadhyay, J. Atulasimha, Complete magnetization reversal in a magnetostrictive nanomagnet with voltage-generated stress: A reliable energy-efficient non-volatile magneto-elastic memory, Applied Physics Letters 105 (7) (2014) 072408. arXiv:http://dx.doi.org/ 10.1063/1.4893617, doi:10.1063/1.4893617. URL http: //dx.doi.org/10.1063/1.4893617

[9] A. K. Biswas, H. Ahmad, J. Atulasimha, S. Bandyopadhyay, Experimental demonstration of complete 180 o reversal of magnetization in isolated co nanomagnets on a pmn-pt substrate with voltage generated strain, Nano Lett. (2017) -doi: 10.1021/acs .nanolett.7b00439.

URL http://pubs.acs.org/doi/abs/10.1021/acs.nanolett. $7 \mathrm{~b} 00439$

[10] J. v. d. Boomgaard, A. M. J. G. van Run, J. Van Suchtelen, Piezoelectricpiezomagnetic composites with magnetoelectric effect, Ferroelectrics 14 (1) (1976) 727-728. doi:10.1080/00150197608236711. URL http://dx.doi.org/10.1080/00150197608236711

[11] K. Roy, S. Bandyopadhyay, J. Atulasimha, Hybrid spintronics and straintronics: A magnetic technology for ultra low energy computing and signal processing, Applied Physics Letters 99 (6) (2011) 063108. arXiv : http: //dx.doi.org/10.1063/1.3624900, doi:10.1063/1.3624900. URL http://dx.doi.org/10.1063/1.3624900

[12] A. K. Biswas, S. Bandyopadhyay, J. Atulasimha, Energy-efficient magnetoelastic non-volatile memory, Applied Physics Letters 104 (23) (2014) 232403. arXiv:http://dx.doi.org/10.1063/1.4882276, doi: $10.1063 / 1.4882276$.

\section{URL http://dx.doi.org/10.1063/1.4882276}

[13] S. Giordano, Y. Dusch, N. Tiercelin, P. Pernod, V. Preobrazhensky, Combined nanomechanical and nanomagnetic analysis of magnetoelectric memories, Phys. Rev. B 85 (15) (2012) 155321-. URL http: //link.aps.org/doi/10.1103/PhysRevB .85.155321

[14] S. Giordano, Y. Dusch, N. Tiercelin, P. Pernod, V. Preobrazhensky, Thermal effects in magnetoelectric memories with stress-mediated switching, Journal of Physics D: Applied Physics 46 (32) (2013) 325002-. URL http: //stacks . iop. org/0022-3727/46/i=32/a=325002

[15] N. Tiercelin, Y. Dusch, S. Giordano, A. Klimov, V. Preobrazhensky, P. Pernod, Nanomagnetic and Spintronic Devices for Energy Efficient Computing, Wiley and Sons., 2015, Ch. 8: Strain Mediated Magnetoelectric Memory.

[16] Z. Zhao, M. Jamali, N. D’Souza, D. Zhang, S. Bandyopadhyay, J. Atulasimha, J.-P. Wang, Giant voltage manipulation of mgo-based magnetic tunnel junctions via localized anisotropic strain: A potential pathway to ultra-energy-efficient memory technology, Applied Physics Letters 109 (9) (2016) 092403. arXiv:http://dx.doi.org/10.1063/ 1.4961670, doi:10.1063/1.4961670.
URL http : //dx . doi.org/10.1063/1.4961670

[17] N. Lei, T. Devolder, G. Agnus, P. Aubert, L. Daniel, J. V. Kim, W. Zhao, T. Trypiniotis, R. P. Cowburn, C. Chappert, D. Ravelosona, P. Lecoeur, Strain-controlled magnetic domain wall propagation in hybrid piezoelectric/ferromagnetic structures, Nature Communications 4 (2013) 1378. arXiv:http://dx.doi.org/10.1038/ncomms2386, doi:10.1038/ ncomms 2386 .

URL http: //dx .doi . org/10.1038/ncomms 2386

[18] K. Roy, Separating read and write units in multiferroic devices, Scientific Reports 5 (2015) 10822. arXiv:http://dx.doi.org/10.1038/ srep10822, doi:10.1038/srep10822.

URL http://dx.doi.org/10.1038/srep 10822

[19] K. Roy, Ultralow energy analog straintronics using multiferroic composites, IEEE Transactions on Nanotechnology 16 (2) (2017) 333 346. arXiv:http://dx.doi.org/10.1109/TNANO.2017.2665481, doi:10.1109/TNANO.2017.2665481.

URL http://dx .doi.org/10.1109/TNANO.2017.2665481

[20] Y. Dusch, V. Rudenko, N. Tiercelin, S. Giordano, V. Preobrazhensky, P. Pernod, Hysteretic magnetoresistance in stress controlled magnetic memory device, Nanomaterials and nanostructures 2 (2012) 44-50.

[21] A. Klimov, N. Tiercelin, Y. Dusch, S. Giordano, T. Mathurin, P. Pernod, V. Preobrazhensky, A. Churbanov, S. Nikitov, Magnetoelectric write and read operations in a stress-mediated multiferroic memory cell, Appl. Phys. Lett. 110 (22) (2017) 222401-. doi:10.1063/1.4983717. URL http://dx.doi.org/10.1063/1.4983717

[22] N. Tiercelin, Y. Dusch, V. Preobrazhensky, P. Pernod, Magnetoelectric memory, granted patent WO 2011/158208

[23] Y. Dusch, N. Tiercelin, A. Klimov, S. Giordano, V. Preobrazhensky, P. Pernod, Stress-mediated magnetoelectric memory effect with uni-axial tbco2/feco multilayer on 011-cut pmn-pt electrostrictive material., J. Appl. Phys. 113 (2013) 17C719. URL http://link. aip.org/link/?JAP/109/07D726/1

[24] N. Tiercelin, V. Preobrazhensky, P. Pernod, A. Ostaschenko, Enhanced magnetoelectric effect in nanostructured magnetostrictive thin film resonant actuator with field induced spin reorientation transition, Appl. Phys. Lett. 92 (6) (2008) 062904-3. URL http://link. aip.org/link/?APL/92/062904/1

[25] N. Tiercelin, A. Talbi, V. Preobrazhensky, P. Pernod, V. Mortet, K. Haenen, A. Soltani, Magnetoelectric effect near spin reorientation transition in giant magnetostrictive-aluminum nitride thin film structure, Appl. Phys. Lett. 93 (16) (2008) 162902-3, Selected for the 2008 november issue of the Virtual Journal of Nanoscale Science \& Technology. URL http: //link. aip.org/link/?APL/93/162902/1

[26] F. Wang, L. Luo, D. Zhou, X. Zhao, H. Luo, Complete set of elastic, dielectric, and piezoelectric constants of orthorhombic $\left(0.71 \mathrm{~Pb}\left(\mathrm{Mg}_{1 / 3} \mathrm{Nb}_{2 / 3}\right) \mathrm{O}_{3}-0.29 \mathrm{PbTiO}_{3}\right)$ single crystal, Appl. Phys. Lett. 90 (21) (2007) 212903-3. URL http://dx .doi.org/10.1063/1.2743393

[27] A. Kákay, L. K. Varga, Monodomain critical radius for soft-magnetic fine particles, JOURNAL OF APPLIED PHYSICS 97 (2005) 083901. arXiv:http://dx.doi.org/10.1063/1.1844612, doi:10.1063/ 1.1844612 . URL http://dx . doi.org/10.1063/1.1844612

[28] S. Giordano, Y. Dusch, N. Tiercelin, P. Pernod, V. Preobrazhensky, Stochastic magnetization dynamics in single domain particles, Eur. Phys. J. B 66 (2013) 249. arXiv:http://dx.doi.org/10.1140/epjb/ e2013-40128-x, doi:10.1140/epjb/e2013-40128-x. URL http://dx.doi.org/10.1140/epjb/e2013-40128-x

[29] J. Z. Sun, Spin-transfer torque switched magnetic tunnel junctions in magnetic random access memory, Proceedings of SPIE 9931 (2016) 113. doi: $10.1117 / 12.2238712$. URL http://dx.doi.org/10.1117/12.2238712

[30] W. Zhao, T. Devolder, Y. Lakys, J. Klein, C. Chappert, P. Mazoyer, Design considerations and strategies for high-reliable stt-mram, Microelectronics Reliability 51 (9-11) (2011) 1454-1458.

URL http://WWW.sciencedirect.com/science/article/pii/ S0026271411002642 